\title{
Pre-clinical evaluation of therapies to prevent or treat bone non-union: a systematic review protocol
}

\author{
Sarah K. Stewart ${ }^{1 *}$, Philippa M. Bennett ${ }^{1}$, Sarah A. Stapley ${ }^{1}$, Janine Dretzke ${ }^{2,3}$, Danai Bem ${ }^{2}$ and \\ Jowan G. Penn-Barwell ${ }^{1}$
}

\begin{abstract}
Background: Non-union of fractured bone is a major cause of morbidity in the orthopaedic population. Despite this, optimal management of non-union is still unclear and remains a significant clinical challenge. Research continues in animal models in an attempt to identify an effective clinical treatment. The proposed systematic review will evaluate current therapies of bone non-union in animal models, in order to identify those that may translate successfully to clinical therapies.

Methods/design: The methodology for the systematic review will be in accordance with standard guidelines. All potential sources for pre-clinical studies will be interrogated and the search strategy written in conjunction with a specialist in this field. Data extraction will be conducted by two reviewers to minimise bias. Analysis will be predominantly qualitative because of the heterogeneity that is likely to exist between the studies. However, quantitative synthesis will be performed where homogeneity in a sub-group of studies exists. Quality assessment will be undertaken utilising a risk of bias tool.

Discussion: To date, there has not been a systematic review addressing bone non-union therapies in animal models despite the plethora of pre-clinical research currently being undertaken. This protocol details and outlines the methodology and justification for such a review.
\end{abstract}

Keywords: Animal model, Pre-clinical trial, Non-union, Delayed union, Critical size defect, Fracture model, Novel therapy

\section{Background}

Non-union is a condition where fractured bone does not heal. This can result in delayed recovery, re-hospitalisation, unplanned revision surgery and potentially, limb amputation $[1,2]$. The rate of non-union can be as high as $30 \%$ in the most severe injuries seen in the civilian setting, rising to $50 \%$ when seen in injuries caused by military weapons [1-3].

A number of therapies including electromagnetic field stimulation, ultrasound, shockwave intervention, and bone morphogenetic protein therapy have been tested in the clinical setting, aimed at preventing or treating non-union. Moreover, they have been evaluated in meta-analysis of clinical trials [4-7]. However, these meta-analyses reveal that there is limited evidence of the efficacy of these

\footnotetext{
* Correspondence: sarah_stewart86@hotmail.com

${ }^{1}$ Royal Centre for Defence Medicine, Queen Elizabeth Hospital, Birmingham B15 2THWest Midlands, UK

Full list of author information is available at the end of the article
}

therapies. Pre-clinical and translational work utilising animal models to develop more potent treatments therefore continues.

Animal models eliminate the confounders that beset clinical work, through standardisation of conditions within a model. However, there is little standardisation between models used by different researchers with a breadth of methodologies and outcome measures being employed to evaluate models of bone non-union [8-11]. Current preclinical work investigating new therapies for promoting bone healing is prolific, as identified in a scoping search of primary studies. Novel strategies that have yet to translate to clinical studies include integrating stem cells into a polymer scaffold embedded with hydroxyapatite particles to stimulate bioactivity [12], use of other growth factors such as stromal-cell derived factor and transforming growth factor to augment the effects of bone morphogenetic proteins [13], and novel synthetic bone substitutes [14].
C Biomed Central

(c) 2015 Stewart et al. Open Access This article is distributed under the terms of the Creative Commons Attribution 4.0 International License (http://creativecommons.org/licenses/by/4.0/), which permits unrestricted use, distribution, and reproduction in any medium, provided you give appropriate credit to the original author(s) and the source, provide a link to the Creative Commons license, and indicate if changes were made. The Creative Commons Public Domain Dedication waiver (http://creativecommons.org/publicdomain/zero/1.0/) applies to the data made available in this article, unless otherwise stated. 
To date, there has been no work to systematically examine and compare all the current pre-clinical and translational evidence to prevent and treat non-union, which is yet to be evaluated in clinical trials. Here, we present a protocol for a systematic review investigating the clinical challenge of bone non-union in animal models.

\section{Objective}

To systematically collect and analyse published and unpublished evidence of therapies for preventing and treating non-union, which are yet to be translated to clinical trials.

\section{Methods}

This protocol was written in accordance with the preferred reporting items for systematic reviews and metaanalysis protocols (PRISMA-P) guidelines [15]. Reporting of the full systematic review will take into account both the PRISMA guidelines [16] and guidelines specific for systematic reviews of animal studies [17]. The protocol has been registered and published on Collaborative Approach to Meta Analysis and Review of Animal Data from Experimental Studies (CAMARADES). The protocol was not registered with PROSPERO, as reviews on animal models were not eligible for inclusion at the time of writing.

\section{Searches}

The following sources will be searched for primary studies:

- Bibliographic databases-PubMed and Embase via Ovid.

- The Science Citation Index for citation searching.

- The British Library's Zetoc research database.

- Hand searching citation lists of included studies and relevant reviews.

- Hand searching of relevant conference proceedings i.e. Orthopaedic Research Society, Orthopaedic Trauma Association, Experimental Biology, Tissue Engineering and Regenerative Medicine Society.

- Contact with study authors and researchers of ongoing trials.

A combination of text and index terms relating to the condition (bone non-union) and population (animal model) will be used. Terms relating to intervention (e.g. stem cells, shock waves) will not be included. This is justified as emerging therapies for bone non-union are likely to be so novel that eligible papers may be missed in the search if intervention terms are included. Although this will undoubtedly produce a high yield of studies from the search, the sensitivity of the search (and therefore comprehensiveness of the systematic review) will be improved.

A filter for the retrieval of animal studies as described by de Vries et al. [18, 19] will be utilised. These have been shown to retrieve greater numbers of animal studies than the standard search filters on these engines. Language restrictions will not be applied. A date restriction (2004-present) will be applied. Justification for this decision stems from the likelihood that studies conducted prior to 2004 will either have already translated to clinical research (rendering them ineligible for inclusion in the systematic review), or found to confer insufficient benefit to warrant translation to the human population. An example search strategy is included in Additional file 1.

An electronic database (EndNote version X7.1, Thomson Reuters, New York) will be used to store and manage search results, regardless of whether the studies are eventually included or excluded in the systematic review. Titles and abstracts generated from the search will be screened by two reviewers (SKS/PMB) in order to establish if they potentially meet the inclusion criteria. Full articles will be obtained for all records that are identified as potentially meeting the inclusion criteria, or where there is uncertainty. Two reviewers (SKS/PMB) will independently screen and evaluate full text reports against the inclusion criteria. Differing of opinions between the two reviewers will be resolved by discussion with a third reviewer (JPB). Where ambiguity remains, contact with the study's authors will be attempted to clarify issues pertaining to eligibility for the systematic review. Papers in languages other than English, whose abstracts indicate that they may meet the inclusion criteria, will be translated in full or in part. A PRISMA flow diagram will be used to document the study selection process, and a record of excluded studies (including reasons for exclusion) will be kept.

\section{Selection criteria \\ Types of studies}

Only controlled trials will be eligible for inclusion. In vitro experiments, cohort studies, case reports, reviews and letters will be excluded. Given the nature and ease of randomisation in animal studies, it is likely that the majority of studies generated from the searches will be randomised controlled trials. Both unpublished and published works are eligible for inclusion.

\section{Types of participants}

Eligible studies must use a mammalian model to test an intervention to treat or prevent bony non-union. There will be no restrictions placed on the anatomical site of the bony defect created. Studies with induced co-morbidities (e.g. immunosuppression, osteoporosis) in the animal subject will be included.

\section{Intervention}

The study assesses an intervention in a mammalian animal model intended to

- Prevent non-union 
- Treat non-union

- Promote or accelerate healing of a bony defect

- Treat or ameliorate delayed union

The intervention must be administered after formation of a bony defect in an animal model. There may be studies that test an established intervention but delivered to the animal model through a novel vehicle. These will be included as they may represent a more effective treatment modality.

\section{Comparator}

A group of control animals are described, which receive

- No treatment

- Current standard of care for the insult in question

- An alternative treatment to the intervention being analysed

\section{Outcome measures}

The primary outcome of interest is bone formation. A quantifiable measure of bone formation must therefore be assessed (morphometrical analysis). This can be achieved through radiological means and/or histological means.

Secondary outcomes of interest will be related to the structural properties of the newly formed bone (biomechanical analysis), which may include:

- Indentation testing

- Microtensile and microcompressive analysis

- Three- and four-point bending

Papers that perform biomechanical analysis (secondary outcomes) in the absence of morphometrical analysis (primary outcomes) will not be included in the systematic review.

\section{Exclusion of therapies with evidence of clinical evaluation}

The stated intention of this study is to examine emerging therapies that have not yet been evaluated clinically. To that end, studies describing therapies that have translated into clinical studies will be filtered. The authors will exclude all papers detailing interventions that they know are already in clinical trials or indeed being used in clinical practice. Where uncertainty exists, the following steps will be performed prior to data extraction:

- PubMed and Embase will be searched for clinical studies that cite each study that otherwise meets the inclusion criteria.

- PubMed and Embase will be searched using keywords describing the specific therapies for studies describing clinical evaluation.

All studies describing therapies that are found to have been evaluated clinically will be excluded, and the results of this 'clinical filter' will added to the PRISMA flow chart.

\section{Data extraction}

A proforma detailing the necessary information to be extracted from the eligible articles has been designed (Additional file 2). The proforma will be piloted by the two reviewers (SKS/PMB) prior to commencing the data collection formally. This will serve to not only test the efficacy of the proforma itself, but also calibrate the reviewers to ensure consistency. Information required (but not limited to) from each study is detailed in Table 1.

\section{Missing data}

During proforma completion, we will seek missing data that is required for analysis from the original study author. If a study does not mention an item detailed on the data collection form and the review authors are unable to obtain the information, its absence will be noted and presumed not to have been performed (e.g. no mention of randomisation will be presumed that it was not performed). This

Table 1 Suggested data to be extracted from eligible papers

\begin{tabular}{ll}
\hline Publication details & Authors, year of study, year of publication, \\
& geographical location of study, language of \\
& paper, funding sources
\end{tabular}

\begin{tabular}{|c|c|}
\hline $\begin{array}{l}\text { Study design and } \\
\text { quality }\end{array}$ & $\begin{array}{l}\text { Sample size, } p \text { values, methods to randomise, } \\
\text { allocation concealment, blinding of } \\
\text { outcome assessment. }\end{array}$ \\
\hline lodel of non-union & Non-union vs delayed union \\
\hline pecies of animal & Mouse, rat, etc. \\
\hline haracteristics of animal & $\begin{array}{l}\text { Age, weight, strain, sex, genetic modification, } \\
\text { co-morbidities }\end{array}$ \\
\hline ature of insult & $\begin{array}{l}\text { Location of bony insult (tibia, femur, etc.), } \\
\text { fracture model (segmental vs non-segmental), } \\
\text { osteotomy technique, size of osteotomy, closed } \\
\text { vs open, critical vs non-critical size defect, } \\
\text { fixation vs no fixation, internal vs external } \\
\text { fixation (if fixation used), isolated or } \\
\text { multiple locations, }\end{array}$ \\
\hline
\end{tabular}

Additional insults (other than bony defects)

Infection, contamination, immunosuppression Nature of intervention

Type of intervention (pharmacological vs non-pharmacological), time point that intervention was applied to animal model post-fracture creation, frequency of intervention (single vs recurrent), duration of intervention, systemic or local dosing

Duration of follow-up Single vs multiple time points of follow-up

Outcome measures Morphometrical analysis (e.g. histology, radiology), biomechanical analysis (e.g. torsional stiffness)

Results and uncertainty, statistical significance, length and adequacy of follow-up, early animal death

Additional insults (other Infection, contamination, immunosuppression, than bony defects) 
domain will be labelled as 'high risk' and its consequential impact on the validity of the study will be assessed when conducting risk of bias assessment (see below).

\section{Quality assessment}

Potential bias within the pre-clinical studies will be determined by the reviewers prior to analysis. Both the Cochrane Collaboration tool [20] and Systematic Review Centre for Laboratory animal Experimentation's (SYRCLE) risk of bias tool [21] which is specific for animal studies will be used. Criteria such as sequence generation, allocation concealment, blinded outcome assessment and incomplete outcome data (e.g. animals that died prematurely) will be identified and recorded, and each domain given a rating of 'high risk' or 'low risk' based on the details provided in the literature. Where information is missing, the domain will be labelled as 'unclear'.

\section{Analysis}

Results from this systematic review will be presented in the form of a narrative synthesis. Key study characteristics such as animal species, intervention and results will be tabulated to aid clarity of study findings.

In the instance where homogeneity exists between a number of studies, quantitative synthesis will be attempted using Review Manager (RevMan v5.3) software. Whilst the generation of pooled effect sizes may not be as meaningful compared to ones provided by clinical trials, meta-analyses of animal studies may still be useful for showing the overall direction of effect and the heterogeneity across studies. Moreover, they may serve to generate hypotheses [22].

\section{Sub-group analysis}

Sub-group analysis will be considered where studies can be grouped according to the following characteristics:

- Animal species (e.g. mice, rats, other mammalian species)

- Animal sex (e.g. male, female, mixed)

- Experimental intervention used (e.g. autografts, allografts, synthetic)

- Delivery of experimental intervention (e.g. oral, intravenously, directly to fracture site).

- Nature of defect created (critical or non-critical defect)

- Models of non-union (hypertrophic or atrophic)

- Models of delayed union versus non-union

- Prevention versus treatment of non-union

- Application of an insult (e.g. osteoporosis, infection, diabetes)

- Methodological quality according to Cochrane risk of bias tool (e.g. by individual domain)

It is recognised that a large number of sub-group analyses on small numbers of studies may affect the power of the analyses; should this be the case here, any findings from sub-group analyses will have to be interpreted cautiously. Presentation of (separate sets of) results in forest plots without the calculation of a summary measure may also be considered in order to highlight any heterogeneity across studies.

\section{Effect measures for continuous outcomes}

The primary outcome measure is bone formation. This will predominantly be in the form of continuous data, for example, bone mass densities $\left(\mathrm{mg} \mathrm{m}^{-2}\right)$. In these instances, the mean difference between formation of bone in the experimental and control groups will likely be presented, and forest plots can be used to show the pooled mean difference. The standardised mean difference may also be used in instances where papers are measuring the same outcome, e.g. bone density, but using different modalities to achieve this, e.g. micro-CT, photon emission CT, and X-ray.

\section{Effect measures for dichotomous outcomes}

In studies which present dichotomous data, the relative risk may be presented and meta-analysis may enable the calculation of a pooled relative risk. A situation where this could occur is analysis of whether bone formation did or did not occur at a set time point. In studies which measure the time to the formation of new bone in the in vivo model, i.e. time-to-event data, the pooled hazard ratio may be calculable.

Given that residual heterogeneity is anticipated even between studies considered to be similar enough to pool, a random effects model (as opposed to a fixed effects model) is more likely to be appropriate for meta-analysis. A random effects model will therefore be used for quantitative analysis of dichotomous data. A random effects model has been chosen because it is based on the assumption that although the studies are different, they are estimating different, yet related intervention effects. If heterogeneity does exist, it will lead to a wider confidence intervals and therefore corresponding claims of statistical significance will be more conservative [23]

A decision on whether to pool will be made on the basis of an assessment of clinical and methodological heterogeneity. However, statistical heterogeneity will also be reported using the $\mathrm{I}^{2}$ statistic and the $\chi^{2}$ test. The likelihood of publication bias will be investigated through the construction of funnel plots for each meta-analysis containing ten or more studies.

\section{Assessment of bias}

Assessing and analysing risk of bias will be an important component of the systematic review. Criteria determining the likelihood of bias will be collected as described above (see 'Quality Assessment') and a 'risk of bias graph' used to 
illustrate where bias predominates across studies. This will serve to inform the authors of where caution must be heeded when analysing the data. It is anticipated that a narrative discussion on the risk of bias will be incorporated into the review, included in which will be discussion on domains which consistently displayed 'low, 'high' or 'unclear' risk. Analysis as to why this may be the case and how this could affect translation of the pre-clinical trial to clinical trials will be discussed.

Where quantitative analysis of subgroups is possible, the threshold based on key bias domains that have been identified during the initial trawl will determine which studies are eligible for inclusion into the subgroup analysis. Key bias domains are derived from the CAMARADES quality checklist for animal studies [24], and include seven domains:

- Publication in peer reviewed journal

- Randomisation of treatment or control

- Allocation concealment

- Blinded assessment of outcome

- Sample-size calculation

- Statement of compliance with regulatory requirements

- Statement regarding possible conflict of interest

It is anticipated that studies at low risk of bias for the key domains will not be combined with studies at high or unclear bias for meta-analysis. In the event where such a priori criteria generate insufficient studies to perform quantitative analysis, pooling of studies regardless of bias risk may have to occur. In this instance, authors will highlight the reasoning for this in the review to ensure transparency of results.

\section{Control group bias}

Due care in data synthesis must also be paid when studies share control groups between experimental groups. For example, a study may have a single control group but three different experimental groups with varying sizes of segmental defects created. When such comparisons are made, animals in the control group are essentially counted three times and the comparisons will receive too much weight in the estimation of summary effect [22]. Techniques such as splitting the control group to equalize the number of experimental groups before performing analysis [23], will be used to mitigate against this potential source of bias.

\section{Discussion}

Systematic reviews of pre-clinical studies enable appraisal and analysis of potential treatments prior to translation to human studies. Although this protocol details the methodology for a systematic review of pre- clinical studies investigating bone non-union, other clinical challenges can use such an approach to the same effect.

In the absence of a widely established, effective treatment for bone non-union, and a lack of systematic reviews of all potentially relevant emerging treatments from the pre-clinical field, a new systematic review is clearly warranted. It is likely that this will be the most comprehensive to date in the area of novel and emerging treatments for bone healing.

A recent review by Mills and Simpson [11] examined the heterogeneity that exists between animal models for non-union, identifying a number of clinical scenarios which animal studies aim to reproduce. Even within subgroups of outwardly similar study designs, the variability between studies was extensive. As such, careful consideration and differentiation of the experimental question of studies included in the review is required. Based on their review, the following recommendations are suggested when sub-grouping papers for analysis.

Of particular note, delayed union is a distinct entity and must be differentiated from non-union in the systematic review to ensure transparency of analysis. Delayed union is prolonged bony healing compared to that of a control group, and will often be the pre-cursor to a non-union model. Unlike a model of non-union where the occurrence of such a defect can be objectively assessed at a set time point, delayed union is a clinical diagnosis with no defined criteria for assessment. Due to the fundamental differences in the definition of delayed union and non-union, separate syntheses will be performed.

Models where a segmental defect has been established should be considered separately to those where there is no iatrogenic bone removal. Attention during data synthesis is also required for models which involve high-energy trauma, open or comminuted fractures, and introduction of infection or immunocompromise, in addition to an intervention intended to treat or prevent non-union. For the purposes of meta-analyses, studies where there has been introduction of such an insult will be analysed separately and not combined with studies where an insult has not been introduced. Inclusion of an insult in an animal model of nonunion will inherently produce higher rates of delayed and non-union, compared with those models where there is an intervention alone.

Finally, it is important to consider the difference between atrophic and hypertrophic models of non-union. Atrophic models are characterised by a failure of periosteal and endosteal activity, with minimal callus formation. Hypertrophic non-union is less common, and is typified by high callus formation and endochondral growth at the fracture sites. Given their contrasting pathophysiological processes, studies with hypertrophic and atrophic models will not be synthesised together. 
As demonstrated in Mills and Simpson's review, the heterogeneic nature of animal models will likely hamper interpretation of results across all studies. Variations in species, methodology and study characteristics may also limit the number of trials considered similar enough to meta-analyse, and sub-group analyses within these sets of studies may also not be possible. However, it is anticipated that by presenting all the available pre-clinical evidence, it will be possible to observe trends for effectiveness for one or more emerging techniques. Pooled effect sizes may not be meaningful in terms of their applicability to human populations, and any findings must be interpreted in the context of study quality and the extent to which they are considered translatable to other settings. However, this systematic review will be valuable for hypothesis generating and may be able to aid in the identification of suitable candidate treatments to take forward into clinical trials. Accurate and valid hypotheses can consequently be drawn as to which emerging therapies for bony non-union may translate successfully to the human population.

\section{Additional files}

Additional file 1: Sample search strategy for PubMed. (DOCX $15 \mathrm{~kb}$ )

Additional file 2: Data collection form. (DOCX $30 \mathrm{~kb}$ )

\section{Abbreviations}

CAMARADES: Collaborative Approach to Meta Analysis and Review of Animal Data from Experimental Studies; PRISMA: Preferred Reporting Items for Systematic Reviews and Meta-Analysis; PRISMA-P: Preferred Reporting Items for Systematic Reviews and Meta-Analysis Protocols; SYRCLE: Systematic Review Centre for Laboratory animal Experimentation.

\section{Competing interests}

The authors declare that they have no competing interests.

\section{Authors' contributions}

SKS drafted and revised the current protocol manuscript and participated in the design of the review and development of search strategies. PMB assisted in revising the protocol manuscript and participated in the design of the review. SAS contributed to the development of the concept of the systematic review, and liaised with the Engineering and Physical Sciences Research Council through the UNIFY programme to secure funding for the review and its protocol. JD contributed to conception and design of the protocol, development of search strategies, and revisions of the protocol. DB contributed to the conception and design of the protocol. JPB was responsible for the original concept of the review, study design, development of search strategies and revisions of the protocol. All authors read and approved the final manuscript.

\section{Acknowledgements}

This manuscript has received funding from a grant awarded by the Engineering and Physical Sciences Research Council through the UNIFY programme.

\footnotetext{
Author details

${ }^{1}$ Royal Centre for Defence Medicine, Queen Elizabeth Hospital, Birmingham B15 2THWest Midlands, UK. ${ }^{2}$ Institute of Applied Health Research, College of Medical and Dental Sciences, University of Birmingham, Edgbaston, Birmingham B15 2TT, UK. ${ }^{3}$ NIHR Surgical Reconstruction \& Microbiology Research Centre, Queen Elizabeth Hospital Birmingham, Edgbaston, Birmingham B15 2TH, UK.
}

Received: 12 August 2015 Accepted: 28 October 2015

Published online: 12 November 2015

\section{References}

1. Harris AM, Althausen PL, Kellam J, Bosse MJ, Castillo RMS, and The Lower Extremity Assessment Project (LEAP) Study Group. Complications following limb-threatening lower extremity trauma. J Orthop Trauma. 2009;23(1):1-6.

2. Penn-Barwell JG, Bennett PM, Fries CA, Kendrew JM, Midwinter MJ, Rickard RF. Severe open tibial fractures in combat trauma: management and preliminary outcomes. Bone Joint J. 2013;95-B(1):101-5.

3. Bennett MH, Stanford RE, Turner R. Hyperbaric oxygen therapy for promoting fracture healing and treating fracture non-union. Cochrane Database Syst Rev. 2012;11:CD004712.

4. Griffin XL, Costa ML, Parsons N, Smith N. Electromagnetic field stimulation for treating delayed union or non-union of long bone fractures in adults. Cochrane Database Syst Rev. 2011;4.

5. Griffin XL, Smith N, Parsons N. Ultrasound and shockwave therapy for acute fractures in adults. Cochrane Database Syst Rev. 2014;6:CD008579.

6. Griffin XL, Wallace D, Parson N, Costa ML. Platelet rich therapies for long bone healing in adults. Cochrane Database Syst Rev. 2012;7:CD009496.

7. Garrison KR, Shemilt I, Donell S, Ryder JJ, Mugford M, Harvey I, et al. Bone morphogenetic protein (BMP) for fracture healing in adults. Cochrane Database Syst Rev. 2010;6:CD006950.

8. Hollinger JO, Kleinschmidt JC. The critical size defect as an experimental model to test bone repair materials. J Craniofac Surg. 1990;1 (1):60-8.

9. Einhorn TA. Clinically applied models of bone regeneration in tissue engineering research. Clin Orthop Relat Res. 1999(367 Suppl): p. S59-67.

10. Histing T, Garcia P, Holstein JH, Klein M, Matthys R, Nuetzi R, et al. Small animal bone healing models: standards, tips, and pitfalls results of a consensus meeting. Bone. 2011;49(4):591-9.

11. Mills LA, Simpson AHRW. In vivo models of bone repair. J Bone Joint Surg, British Volume. 2012;94-B(7):865-74.

12. Tayton E, Purcell M, Smith JO, Lanham S, Howdle SM, Shakesheff KM, et al. The scale-up of a tissue engineered porous hydroxyapatite polymer composite scaffold for use in bone repair: an ovine femoral condyle defect study. J Biomed Mater Res A. 2015;103(4):1346-56.

13. Herberg S,Kondrikova G, Periyasamy-Thandavan S, Howie RN, Elsalanty ME, Weiss L, et al. Inkjet-based biopatterning of SDF-1 beta augments BMP2 -induced repair of critical size calvarial bone defects in mice. Bone. 2014;67:95-103.

14. Calvo-Guirado JL, Delgado-Ruíz RA, Ramírez-Fernández MP, Maté-Sánchez JE, Ortiz-Ruiz A, Marcus A. Histomorphometric and mineral degradation study of Ossceram: a novel biphasic B-tricalcium phosphate, in critical size defects in rabbits. Clin Oral Implants Res. 2012;23(6):667-75.

15. Shamseer L, Moher D, Clarke M, Ghersi D, Liberati A, Petticrew M, et al. Preferred reporting items for systematic review and meta-analysis protocols (PRISMA-P) 2015: elaboration and explanation. BMJ. 2015;349:97647.

16. Moher D, Liberati A, Tetzlaff J, Altman DG; PRISMA Group. Preferred reporting items for systematic reviews and meta-analyses: the PRISMA statement. BMJ. 2009;339:b2535.

17. Sena ES, Currie GL, McCann SK, Macleod MR, Howells DW. Systematic reviews and meta-analysis of preclinical studies: why perform them and how to appraise them critically. J Cereb Blood Flow Metab. 2014;34(5):737-42.

18. de Vries RBM , Hooijmans CR, Tillema A, Leenaars M, Ritskes-Hoitinga M. A search filter for increasing the retrieval of animal studies in Embase. Lab Anim. 2011;45(4):268-70.

19. Hooijmans CR, Tillema A, Leenaars M, Ritskes-Hoitinga M. Enhancing search efficiency by means of a search filter for finding all studies on animal experimentation in PubMed. Lab Anim. 2010;44(3):170-5.

20. Higgins JPT,Altman DG, Gøtzsche PC, Jüni P, Moher D, Oxman AD, et al. The Cochrane Collaboration's tool for assessing risk of bias in randomised trials. BMJ 2011;343:d5928.

21. Hooijmans CR, Rovers MM, de Vries RB, Leenaars M, Ritskes-Hoitinga M, Langendam MW. SYRCLE's risk of bias tool for animal studies. BMC Med Res Methodol. 2014;14:43.

22. Hooijmans CR, IntHout J, Ritskes-Hoitinga M, Rovers MM. Meta-analyses of animal studies: an introduction of a valuable instrument to further improve healthcare. ILAR J. 2014;55(3):418-26.

23. Higgins J, Deeks J, Altman D. Special topics in statistics. In: Higgins JPT, Green S, editors. Cochrane Handbook for Systematic Reviews of Interventions. John Wiley \& Sons Ltd, Chichester. 2008

24. Sena E, van der Worp HB, Howells D, Macleod M. How can we improve the pre-clinical development of drugs for stroke? Trends Neurosci. 2007;30(9):433-9. 